\title{
Calcineurin inhibitors in bronchiolitis obliterans syndrome following stem cell transplantation
}

\author{
Katrin E. Hostettler, ${ }^{1,2}$, Jörg P. Halter ${ }^{3}$, Sabine Gerull ${ }^{3}$, Didier Lardinois ${ }^{4}$, \\ Spasenija Savic ${ }^{5}$, Michael Roth ${ }^{1}$ and Michael Tamm ${ }^{1,2}$
}

\author{
Affiliations: \\ 'Dept of Biomedicine, University Hospital Basel, Basel, \\ ${ }^{2}$ Clinic of Respiratory Medicine, University Hospital Basel, Basel, \\ ${ }^{3}$ Clinic of Hematology, University Hospital Basel, Basel, \\ ${ }^{4}$ Clinic of Thoracic Surgery, University Hospital Basel, Basel, and \\ ${ }^{5}$ Institute for Pathology, University Hospital Basel, Basel, Switzerland.
}

Correspondence: K.E. Hostettler, Clinic of Respiratory Medicine, University Hospital Basel, Petersgraben 4, 4031 Basel, Switzerland. E-mail: katrin.hostettlerQusb.ch

ABSTRACT Bronchiolitis obliterans is a complication after allogeneic haematopoietic stem cell transplantation (HSCT). Management of bronchiolitis obliterans comprises intensive immunosuppression, but treatment response is poor. We investigated the effect of cyclosporine A (CsA), tacrolimus (FK506), methylprednisolone (mPRED), mycophenolate mofetil (MMF) and everolimus on the proliferation of primary lung myofibroblasts from HSCT patients with bronchiolitis obliterans syndrome (BOS).

Cells were isolated from surgical lung biopsies of eight HSCT patients with BOS. Proliferation was assessed by $\left[{ }^{3} \mathrm{H}\right]$-thymidine incorporation.

Biopsies revealed constrictive bronchiolitis obliterans in three patients and lymphocytic bronchiolitis in five patients. CsA and FK506 significantly induced proliferation of myofibroblasts. mPRED and MMF caused a significant inhibition of proliferation, whereas everolimus had no effect. Costimulation with FK506, mPRED and MMF significantly inhibited proliferation. Serial pulmonary function tests over 12 months after lung biopsy and under triple therapy demonstrated that patients with lymphocytic bronchiolitis had a significant improvement in forced expiratory volume in 1 s (FEV1), whereas FEV1 of patients with bronchiolitis obliterans was unchanged.

Our data demonstrate a pro-proliferative effect of calcineurin inhibitors on primary human lung myofibroblasts obtained from patients with BOS after HSCT. In contrast, based on the observed antiproliferative capacity of MMF in vitro, MMF-based calcineurin inhibitor-free treatment strategies should be further evaluated in patients with bronchiolitis obliterans after HSCT.

@ERSpublications

Calcineurin inhibitor-free regimens need further evaluation in bronchiolitis obliterans after stem cell transplantation http://ow.ly/qeKha 


\section{Introduction}

Allogeneic haematopoietic stem cell transplantation (HSCT) is an established treatment option for several malignant and nonmalignant disorders [1]. However, long-term survival is limited by recurrence of the underlying disease and development of chronic graft-versus-host disease (GvHD), the most common late complication after allogeneic HSCT [2]. The manifestation of chronic GvHD in the lungs, bronchiolitis obliterans [3], has a reported incidence of $5-20 \%[4,5]$. Bronchiolitis obliterans is characterised by the new development of fixed airflow obstruction, manifesting with cough, dyspnoea and wheezing $[3,6]$. The term bronchiolitis obliterans stands for a histopathologically proven diagnosis, showing damage to the bronchiolar epithelium, obliteration of bronchiolar lumens and inflammation between the epithelium and smooth muscle [7]. In the absence of a histological confirmation, the clinical diagnosis of bronchiolitis obliterans syndrome (BOS) requires decreased airflow in pulmonary function tests and air trapping in computed tomography (CT) scans $[3,8]$.

Even though bronchiolitis obliterans is strongly associated with chronic GvHD [9], the heterogeneous histopathological findings and clinical course imply that formation of bronchiolitis obliterans is a multifactorial process involving both alloimmune and nonalloimmune mechanisms [10]. The pathogenesis of bronchiolitis obliterans is believed to be initiated by repetitive lung tissue injuries. Those injuries may be caused by different factors, such as the interaction between donor T-lymphocytes and recipient epithelial cells [11], tissue damage caused by the conditioning regimen [12] or infection [13]. Whether or not immunosuppressive agents play a role in bronchiolitis obliterans development and/or progression after HSCT, beyond an increased susceptibility to infections, has not yet been studied.

Data from lung transplantation literature suggest that immunosuppressive agents such as calcineurin inhibitors and corticosteroids are probably beneficial by slowing down the progression of BOS [14, 15]. Therefore, treatment for bronchiolitis obliterans after HSCT is based on reinstitution or augmentation of immunosuppressive therapy with high-dose systemic corticosteroids and other agents, namely cyclosporine A (CsA) and tacrolimus (FK506) [16, 17]. However, despite different treatment approaches, prognosis of bronchiolitis obliterans remains poor, with an overall 3-year mortality of up to 65\% [18]. In addition, the potential benefit of augmented immunosuppression might be limited due to the occurrence of lifethreatening pulmonary infections. Currently, no data from large controlled trials are available, and bronchiolitis obliterans treatment is based on expert opinion and small case series only.

The fibrogenic effects of CsA and FK506 have been studied extensively in renal allografts [19], and are predominantly mediated by elevated expression of transforming growth factor- $\beta[20,21]$. In addition, previous data have demonstrated that CsA may directly stimulate cell proliferation [22, 23], raising the possibility that calcineurin inhibitors may contribute to the development of chronic GvHD in the lung. The aim of the present study was to investigate the in vitro effect of the immunosuppressive agents CsA, FK506, methylprednisolone (mPRED), mycophenolate mofetil (MMF) and everolimus on the proliferative capacity of primary human lung cells obtained from patients with BOS after HSCT.

\section{Materials and methods}

Patients

Primary human lung cell cultures were successfully established from lung tissue obtained from surgical lung biopsies of eight patients with BOS after HSCT. All patients had an irreversible decline in forced expiratory volume in $1 \mathrm{~s}$ (FEV1) of $>15 \%$ and symptoms suggestive of bronchiolitis obliterans, and thus underwent surgical lung biopsy for final diagnosis. Before lung biopsy all patients had a high-resolution CT scan and underwent flexible bronchoscopy with bronchoalveolar lavage (BAL) to exclude infection as a cause of lung function decline. CT scans were analysed for pulmonary infiltrates, bronchial wall thickening, bronchiectasis and air trapping. Total and differential lavage fluid cell counts were performed. Lung biopsy was performed by video-assisted thoracoscopic surgery (VATS). Lung wedges were inflated and fixed with $4 \%$ buffered formalin. After paraffin embedding, specimens were cut into $4-\mu \mathrm{m}$ sections and routinely stained with haematoxylin and eosin, elastica van Gieson, alcian blue-periodic acid Schiff, and Grocott's methenamine silver stain. Tissue sections were reviewed by two experienced lung pathologists. Patient characteristics are shown in table 1 . All patients are part of a larger cohort, the clinical characteristics of which are described in more detail in a separate paper [24].

\section{Pulmonary function tests}

Patients underwent a clinical assessment and a complete set of pulmonary function tests before and after HSCT, before VATS, and 3, 6 and 12 months after VATS. Pulmonary function tests were performed using body plethysmography and carbon monoxide diffusion capacity (Jaeger, Wuerzburg, Germany). All testing 


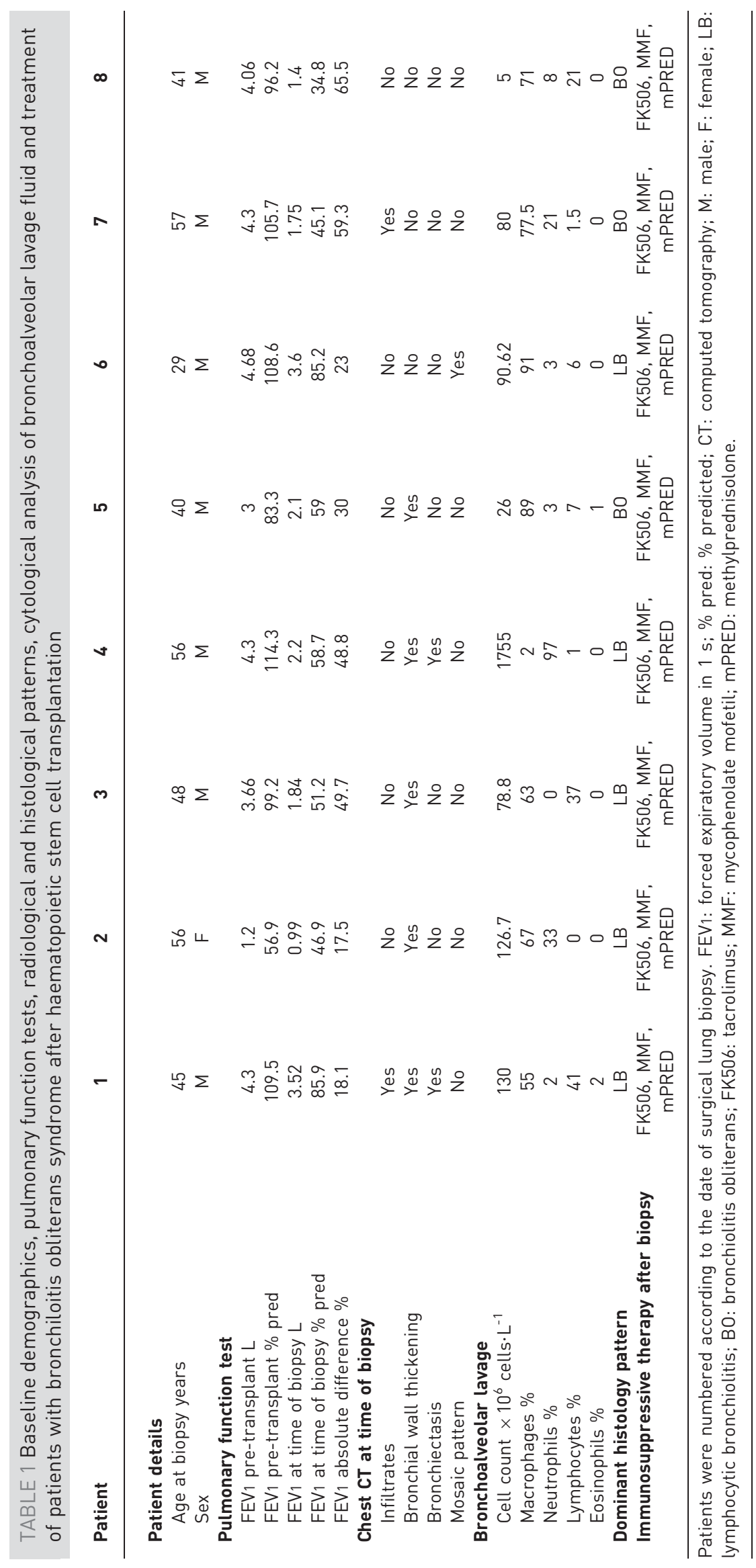


was performed according to European Respiratory Society standards [25]. Pulmonary function follow-up data were available from seven patients; one patient died due to severe infection 4 months after diagnosis of BOS.

\section{Cell culture}

Human lung tissue was obtained with approval of the human ethics committee of the University of Basel (Basel, Switzerland) and written informed consent was obtained from all patients who underwent surgical lung biopsy. Primary human lung cell cultures were established as reported previously [26]. Briefly, fresh peripheral lung tissue was obtained from the lung of patients undergoing diagnostic resection, and was cut into small pieces of $1 \mathrm{~mm}^{3}$ which were placed into pre-wetted $25-\mathrm{cm}^{2}$ cell culture flasks. Culture medium, consisting of RPMI (Gibco Invitrogen, Lucerne, Switzerland) supplemented with $10 \%$ fetal calf serum (FCS) (Gibco), $20 \mathrm{U} \cdot \mathrm{L}^{-1}$ penicillin, $20 \mu \mathrm{g} \cdot \mathrm{mL}^{-1}$ streptomycin and $2.5 \mu \mathrm{g} \cdot \mathrm{mL}^{-1}$ amphotericin B (Gibco), was replaced every fourth day. Cells were grown under standard conditions $\left(37^{\circ} \mathrm{C}, 21 \% \mathrm{O}_{2}, 5 \% \mathrm{CO}_{2}\right)$. At $90 \%$ confluence the cell layer was trypsinised with a solution of $0.5 \%$ trypsin in $1 \mathrm{mM}$ disodiumEDTA. All experiments were performed using cells at passage three to six.

\section{Immunofluorescence analysis}

For immunofluorescence analysis cells were plated in six-well plates and grown in RPMI, 10\% FCS. Cells were fixed with $4 \%$ formalin (10 $\mathrm{min}$ ) and permeabilised with acetic acid (3:1, ice-cold, $10 \mathrm{~min})$. Cells were then blocked with $5 \%$ bovine serum albumin $(1 \mathrm{~h})$ and incubated with antibodies against fibronectin (Santa Cruz, LabForce AG, Nunningen, Switzerland), $\alpha$-smooth muscle actin (Epitomics, LabForce AG, Nunningen, Switzerland) and cofilin (Santa Cruz) for $1 \mathrm{~h}$. The primary antibodies were detected by addition of fluorescein-conjugated goat anti-rabbit (Bethyl Laboratories, Lubio Science, Lucerne, Switzerland), fluorescein-conjugated donkey anti-goat (Southern Biotech, BioConcept, Allschwil, Switzerland) or cy3conjugated goat anti-rabbit antibodies (Invitrogen; Lucerne, Switzerland) for cofilin, fibronectin and $\alpha$-smooth muscle actin, respectively, for $1 \mathrm{~h}$. To visualise the nuclei, 4,6-diamido-2-phenylindole dihydrochloride (Sigma-Aldrich, Buchs, Switzerland) was added (5 min) and the well-plates were subsequently examined on a fluorescence microscope.

\section{Proliferation assay}

Primary human lung myofibroblasts were seeded onto a 96-well plate at a density of $1 \times 10^{5} \mathrm{cells} \cdot \mathrm{mL}^{-1}$ and were grown until 60-70\% confluent in growth medium. Cells were rinsed twice with PBS, and were then serum-starved for $24 \mathrm{~h}$. Myofibroblasts were incubated in RPMI with or without drug (control). The effect of mPRED (methylprednisolone; Sigma-Aldrich), CsA (Sandimmune; Novartis, Basel, Switzerland), FK506 (Prograf; Fujisawa, Osaka, Japan), everolimus (Sigma-Aldrich) and MMF (in all experiments mycophenolic acid was used, which is the biologically active form of MMF) (mycophenolic acid, Sigma-Aldrich) were assessed. Myofibroblasts incubated with RPMI supplemented with 10\% FCS were used as a control for proliferative response. After $19 \mathrm{~h} 1 \mu \mathrm{Ci}\left[{ }^{3} \mathrm{H}\right]$-thymidine (PerkinElmer, Zaventem, Belgium) in $10 \mu \mathrm{L}$ of RPMI was added to each well. After a further 5-h incubation period, $100 \mu \mathrm{L}$ of $1 \mathrm{M} \mathrm{NaOH}$ was added to each well and cells were then harvested onto filter plates (PerkinElmer) using the Filtermate Unifilter-96 Harvester (PerkinElmer). The filter plates were then dried overnight at room temperature before addition of $20 \mu \mathrm{L}$ of scintillation fluid (PerkinElmer) to each well. Counts per minute were obtained on a TopCount Microplate scintillation counter (PerkinElmer) for 1 min per sample.

\section{Statistical analysis}

Statistical comparisons were made by using paired t-tests for paired observations. $p \leqslant 0.05$ was considered significant. Where applicable, data are shown as mean \pm SEM from at least three independent experiments.

\section{Results}

The characteristics of the eight patients are shown in table 1. The mean (range) decline of absolute FEV1 between pre-transplant (stable baseline) and lung biopsy was -39\% (-17.5--65.5\%) (table 1). Radiological changes on high-resolution chest CT scans with focus on signs of air-trapping and bronchiectasis are summarised in table 1 . Three patients had elevated numbers of neutrophils in their BAL fluid and another three had a high proportion of lymphocytes. No patient had concomitant infection. Lung biopsy tissue revealed typical bronchiolitis obliterans in three patients and lymphocytic bronchiolitis in five patients (table 1). Representative lung tissue sections of bronchiolitis obliterans and lymphocytic bronchiolitis are shown in figure 1. Post-biopsy BOS treatment comprised a combination of steroids, FK506 and MMF in all patients (table 1).

As shown in figure 2, the cultured primary lung cells exhibited typical spindle-shape morphology of mesenchymal cells, and immunofluorescence studies demonstrated positive immunoreaction for both 

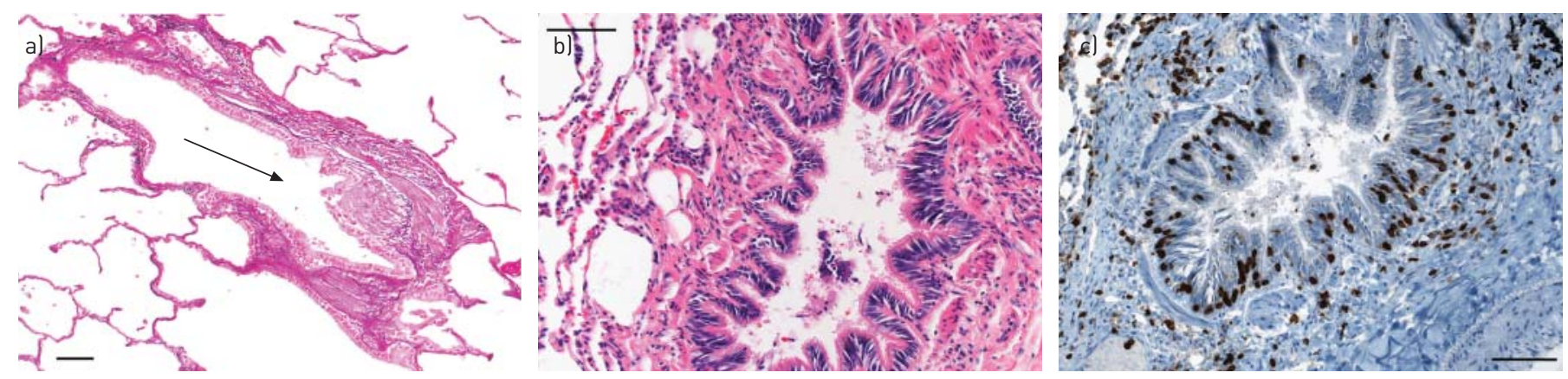

FIGURE 1 a) Representative histological section of a lung with bronchiolitis obliterans showing excentric, subepithelial connective tissue depositions (arrow) in the absence of inflammation. Elastica van Gieson staining. b) Representative histological section of a lung with lymphocytic bronchiolitis demonstrating an intraepithelial mononuclear inflammatory infiltrate and lack of luminal obliteration. Haematoxylin and eosin staining. c) Representative lung section of lymphocytic bronchiolitis that highlights the presence of intraepithelial CD8 lymphocytes. CD8 immunohistological staining. Scale bars $=100 \mu \mathrm{m}$.

fibronectin (fig. 2b) and $\alpha$-smooth muscle actin (fig. 2d), identifying the cells as myofibroblasts. This was further confirmed by positive immunoreaction for the typical myofibroblast marker cofilin (fig. 2f). Cells kept these characteristics over several culture passages.

Proliferation of myofibroblasts after growth stimulation with 10\% FCS was increased fourfold (406 $\pm 115 \%$ ) compared with cells grown in FCS-free medium (fig. 3). We found no significant difference between baseline (FCS-free) and 10\% FCS-stimulated proliferative capacity of myofibroblasts derived from patients with histologically confirmed bronchiolitis obliterans compared with those obtained from patients with lymphocytic bronchiolitis.

Low concentrations of CsA $\left(0.01 \mathrm{mg} \cdot \mathrm{L}^{-1}\right.$ and $\left.0.1 \mathrm{mg} \cdot \mathrm{L}^{-1}\right)$ significantly induced cell proliferation compared to untreated cells (fig. 4a). CsA at $0.01 \mathrm{mg} \cdot \mathrm{L}^{-1}$ caused a $39.9 \pm 11.9 \%$ induction of cell growth compared with untreated controls $(\mathrm{n}=8, \mathrm{p}<0.05)$ (fig. $4 \mathrm{a})$ and $0.1 \mathrm{mg} \cdot \mathrm{L}^{-1}$ CsA had a similar stimulatory effect with $32.7 \pm 12.6 \%$ induction of cell growth $(\mathrm{n}=8, \mathrm{p}<0.05)$ (fig $4 \mathrm{a})$. Only high-dose CsA $\left(50 \mathrm{mg} \cdot \mathrm{L}^{-1}\right)$ exerted a significant antiproliferative effect of $56 \pm 14.3 \%$ growth reduction $(n=8, p<0.05)$ (fig. $4 a)$. However, this effect was accompanied by a decreased viability of cells (trypan blue exclusion), as reported earlier [27]. Likewise, FK506 caused an induction of cell proliferation at low concentrations (fig. 4b) at $0.001 \mathrm{mg} \cdot \mathrm{L}^{-1}$ FK506 caused a $37.6 \pm 15.1 \%$ induction of cell growth $(\mathrm{n}=8, \mathrm{p}<0.05)$ (fig. $4 \mathrm{~b}$ ), at $0.01 \mathrm{mg} \cdot \mathrm{L}^{-1}$ a $38.3 \pm 15.5 \%$ induction of cell growth $(\mathrm{n}=8, \mathrm{p}<0.05)$ (fig. $4 \mathrm{~b})$ and at $0.1 \mathrm{mg} \cdot \mathrm{L}^{-1} \mathrm{FK} 506 \mathrm{had}$ a $27.0 \pm 12.2 \%$ stimulatory effect on cell proliferation $(n=8, p<0.05)$ (fig. $4 b)$. A significant antiproliferative effect of FK506 was observed only at a concentration of $5 \mathrm{mg} \cdot \mathrm{L}^{-1}(27 \pm 15.4 \%$ inhibition; $\mathrm{n}=8, \mathrm{p}<0.05)$ (fig. 4b), but again combined with decreased cell viability. mPRED had no growth-stimulatory effect at any concentration, but caused a significant inhibition of proliferation at concentrations of $10 \mathrm{mg} \cdot \mathrm{L}^{-1}$ $(14.8 \pm 4.9 \%$ inhibition; $\mathrm{n}=8, \mathrm{p}<0.05)$ (fig. $4 \mathrm{c}), 50 \mathrm{mg} \cdot \mathrm{L}^{-1}(18.2 \pm 7.5 \%$ inhibition; $\mathrm{n}=8, \mathrm{p}<0.05)$ (fig. 4c) and $100 \mathrm{mg} \cdot \mathrm{L}^{-1}(35.7 \pm 7.5 \%$ inhibition, $\mathrm{n}=8, \mathrm{p}<0.05)$ (fig. $\left.4 \mathrm{c}\right)$. Similarly, MMF caused a significant inhibition of proliferation at concentrations of $0.01 \mathrm{mg} \cdot \mathrm{L}^{-1}(17.9 \pm 3.4 \%$ inhibition; $\mathrm{p}<0.05)$ (fig. $4 \mathrm{~d}$ ) and $1 \mathrm{mg} \cdot \mathrm{L}^{-1} \quad(25.6 \pm 9.0 \%$ inhibition; $\mathrm{p}<0.05) \quad$ (fig. $\left.4 \mathrm{~d}\right)$. Everolimus had no significant effect on cell proliferation at any concentration tested $(n=8)$ (fig 4 e).

There was no difference in proliferative responses after drug treatment when cell lines were grouped according to histological findings: cell lines derived from bronchiolitis obliterans lungs did not differ in proliferative response from cell lines derived from lymphocytic bronchiolitis lungs (data not shown).

According to the administered BOS treatment (table 1), myofibroblasts obtained from patients with BOS after HSCT were stimulated with the combination of FK506 $\left(0.01 \mathrm{mg} \cdot \mathrm{L}^{-1}\right), \mathrm{mPRED}\left(10 \mathrm{mg} \cdot \mathrm{L}^{-1}\right)$ and MMF $\left(1 \mathrm{mg} \cdot \mathrm{L}^{-1}\right)$. As shown in figure 5 , this triple treatment caused a significant inhibition of cell proliferation $(33 \pm 6.9 \%$ inhibition, $\mathrm{p}<0.05)$ (fig. 5), which was more pronounced than with mPRED alone at the same concentration. To study the MMF portion of the antiproliferative effect, myofibroblasts were stimulated with MMF alone, and this caused a similar inhibitory effect as the triple stimulation $(40.2 \pm 5.0 \%$ inhibition, $\mathrm{p}<0.05$ ) (fig. 5).

Follow-up pulmonary function tests were performed at 3,6 and 12 months after lung biopsy. Figure 6a depicts the course of FEV1 (\% predicted) of all patients from the time point of VATS, and 3, 6 and 12 months after lung biopsy. Figure $6 \mathrm{~b}$ shows the development of mean FEV1 \% pred for the two groups. Compared to pre-lung-biopsy values, patients with lymphocytic bronchiolitis had a statistically significant 

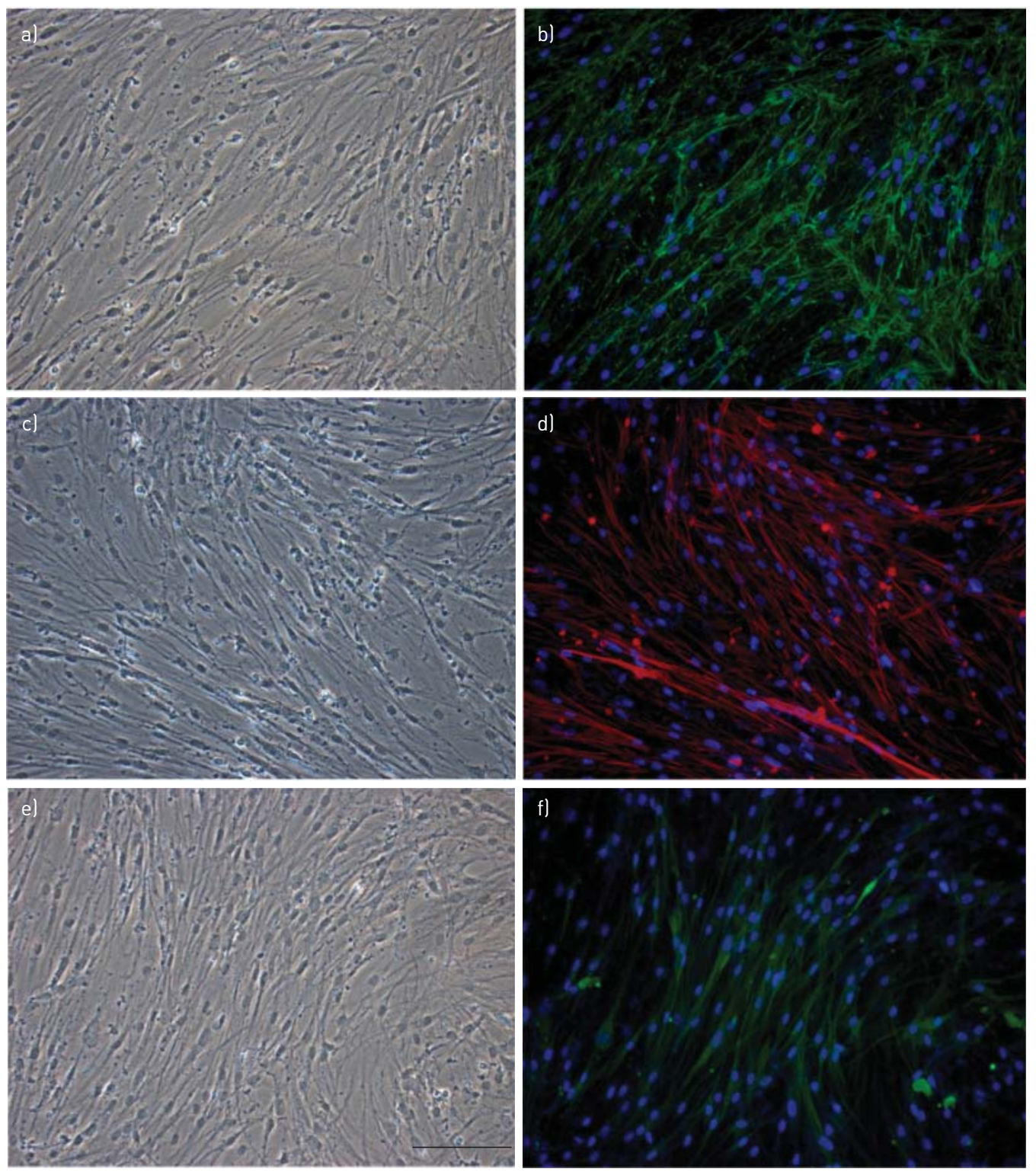

FIGURE 2 Primary human lung cells immunostained for b) fibronectin, d) $\alpha$-smooth muscle actin and f) cofilin. Corresponding phase contrast pictures are shown in panels a), c) and e). Cells were grown to confluence in normal growth medium, were then fixed and permeabilised. Primary antibodies were detected by addition of fluorescein-labelled (green) or Cy3-labelled (red) secondary antibodies. Visualisation by fluorescence microscopy. Scale bar $=200 \mu \mathrm{m}$.

mean improvement in FEV1 \% pred after 3 months $(+17.3 \%, n=5, p \leqslant 0.05)$ (fig. 6b), 6 months (+30.9\%, $\mathrm{n}=4, \mathrm{p} \leqslant 0.05$ ) (fig $6 \mathrm{~b}$ ) and after 12 months $(+29.2 \%, \mathrm{n}=4, \mathrm{p} \leqslant 0.05)$ (fig. $6 \mathrm{~b}$ ), whereas patients with histological bronchiolitis obliterans showed no significant change in FEV1 \% pred.

\section{Discussion}

In this study we investigated the in vitro effect of immunosuppressive agents on the proliferative capacity of primary human lung myofibroblasts obtained from patients with BOS after allogeneic HSCT. We found that the calcineurin inhibitors CsA and FK506 in clinically relevant concentrations (CsA $0.01 \mathrm{mg} \cdot \mathrm{L}^{-1}$ and $0.1 \mathrm{mg} \cdot \mathrm{L}^{-1}$; and FK506 $0.001 \mathrm{mg} \cdot \mathrm{L}^{-1}, 0.01 \mathrm{mg} \cdot \mathrm{L}^{-1}$ and $\left.0.1 \mathrm{mg} \cdot \mathrm{L}^{-1}\right)$ enhanced proliferation of myofibroblasts, whereas MMF and mPRED caused a significant inhibition of cell proliferation. Finally, combination therapy of FK506, MMF and mPRED, according to the treatment for BOS administered in our study group, caused a significant inhibition of myofibroblast proliferation. 

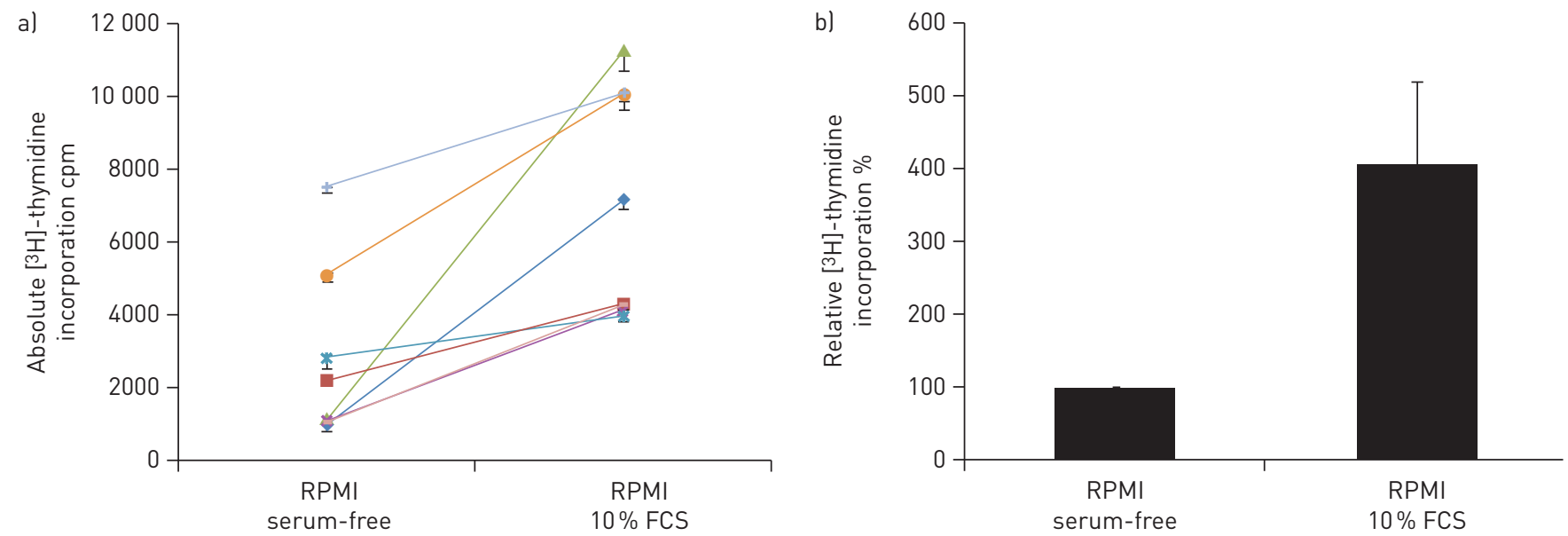

FIGURE 3 Effect of $10 \%$ fetal calf serum (FCS) growth medium on myofibroblast proliferation compared with serum-free medium. Primary human lung myofibroblasts $(\mathrm{n}=8)$ were incubated for $24 \mathrm{~h}$ with serum-free RPMI medium or with RPMI containing $10 \%$ FCS. Cell growth was assessed by [ $\left.{ }^{3} \mathrm{H}\right]$-thymidine incorporation. a) Absolute values for each tested cell line; b) mean \pm SEM values of eight independent experiments, expressed as relative proliferation compared with control (serum-free RPMI). Each experiment was performed in triplicate. cpm: counts per minute.

Surgical lung biopsy remains the gold standard for diagnosis of bronchiolitis obliterans [3]. However, surgical lung biopsy is often avoided, due to potential high morbidity and mortality in this patient group. In a study by SANTO TOMAS et al. [28] only 13\% of patients with suspicion of bronchiolitis obliterans underwent surgical lung biopsy to attempt histological confirmation, and thus only limited data on the histological correlate of BOS is available. Among our eight patients with BOS, only three (38\%) showed a typical constrictive bronchiolitis obliterans pattern on lung biopsy. The other five patients showed the histological pattern of lymphocytic bronchiolitis with a prominent lymphocytic infiltration of the bronchiolar wall with variable epithelial inflammation and damage. The finding that approximately a third of patients with BOS exhibit the classic histological pattern of constrictive bronchiolitis obliterans is supported by the data of a larger series of HSCT patients with BOS who underwent surgical lung biopsy [24]. Lymphocytic bronchiolitis has been categorised as a variant of lung GvHD [16, 29]. However, the current standard of knowledge does not allow the decision on whether lymphocytic bronchiolitis and bronchiolitis obliterans are two independent processes, or whether lymphocytic bronchiolitis and bronchiolitis obliterans represent an early (lymphocytic bronchiolitis) and a later (bronchiolitis obliterans) stage of the same disease process, i.e. chronic lung GvHD. The hypothesis that the fibrotic process observed in bronchiolitis obliterans is preceded by an inflammatory phase (lymphocytic bronchiolitis) is supported by the observations in a rat model of bronchiolitis obliterans following lung transplantation where lymphocytic infiltration was a precursor of fibrous obliteration [30]. Furthermore, on the basis of a patient case with slowly progressive BOS, it has been demonstrated recently that lymphocyte infiltration in the bronchial walls without fibrosis preceded the typical pathological findings of bronchiolitis obliterans [31].

Irrespective of the histological pattern, augmented immunosuppressive therapy, including calcineurin inhibitors, is widely used for the treatment of BOS after HSCT. However, to date a beneficial effect of the calcineurin inhibitor treatment for the course of BOS/bronchiolitis obliterans after HSCT has not been demonstrated. CsA and FK506 both inhibit calcineurin, which is responsible for calcium-dependent signal transduction, thereby preventing the transcription of lymphokine genes, such as interleukin-2, and inhibiting T-cell activation and proliferation [32]. However, a fibrogenic effect of CsA and FK506 has been described earlier in renal allografts and in animal and in vitro models [19-21]. The fibrogenic effects are predominantly mediated by elevated expression of transforming growth factor- $\beta$, suppressed matrix metalloproteinase activity and augmented fibroblast collagen synthesis [20, 21]. To our knowledge, we are the first to culture primary human lung cells derived from HSCT patients with BOS, and by using these cells we were able to demonstrate that CsA and FK506 at clinically relevant immunosuppressive concentrations have a significant pro-proliferative effect on primary human lung myofibroblasts. The concentrations at which this effect was observed lay between $0.001 \mathrm{mg} \cdot \mathrm{L}^{-1}$ and $1 \mathrm{mg} \cdot \mathrm{L}^{-1}$ for CsA and between 0.001 and $0.01 \mathrm{mg} \cdot \mathrm{L}^{-1}$ for FK506, and thus correspond to target whole-blood concentrations of $0.15-0.25 \mathrm{mg} \cdot \mathrm{L}^{-1}$ for CsA and $0.01-0.02 \mathrm{mg} \cdot \mathrm{L}^{-1}$ for FK506 [33]. Our in vitro data showing a pro-proliferative effect of calcineurin inhibitors in primary human lung cells are in line with data obtained in other cell types [22, 23], and correspond to the observed pro-fibrotic effect in vivo [34]. Only at very high concentrations did both CsA $\left(50 \mathrm{mg} \cdot \mathrm{L}^{-1}\right)$ and FK506 $\left(5 \mathrm{mg} \cdot \mathrm{L}^{-1}\right)$ exert a significant anti-proliferative effect on primary human lung 

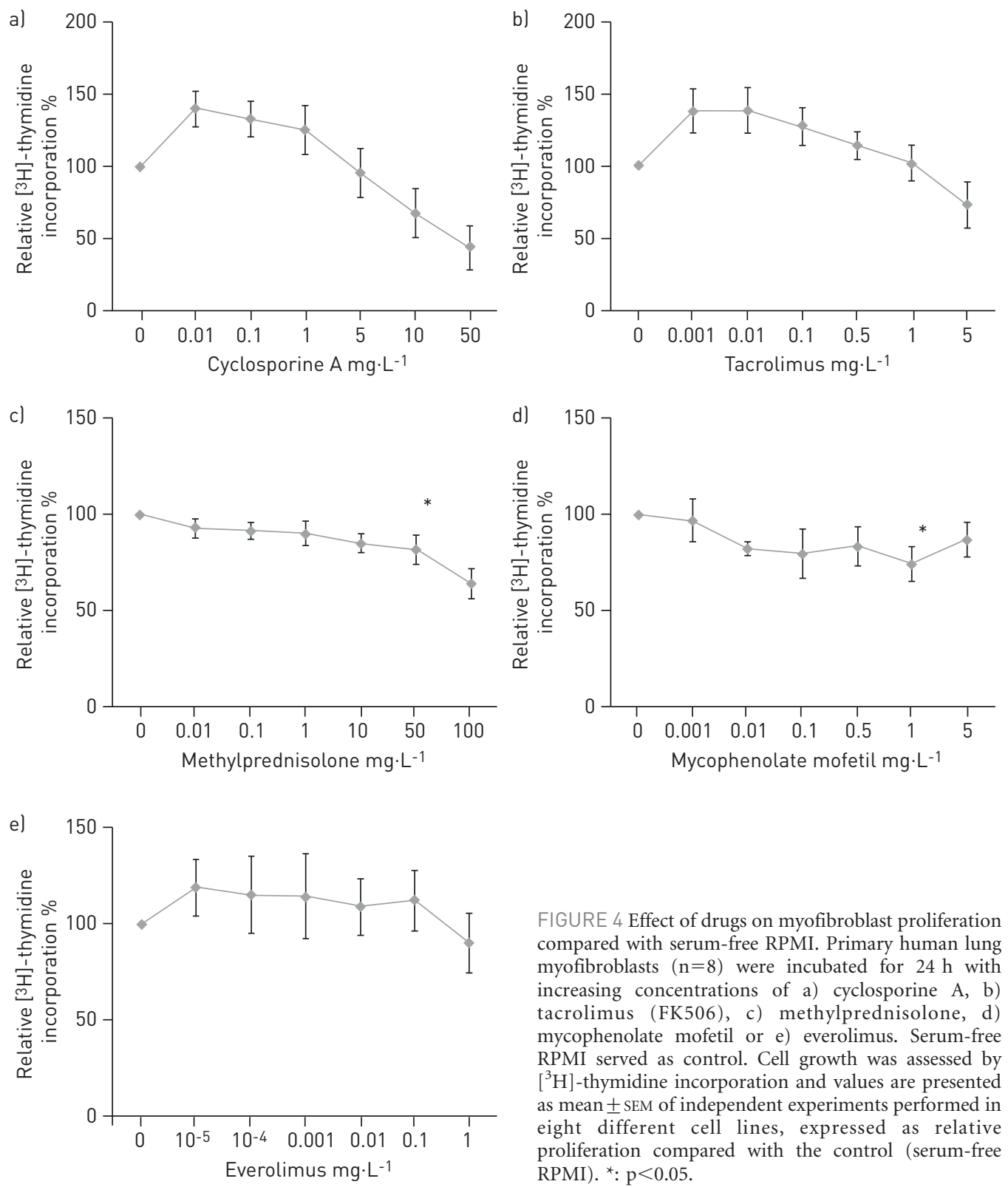

FIGURE 4 Effect of drugs on myofibroblast proliferation
compared with serum-free RPMI. Primary human lung
myofibroblasts $(\mathrm{n}=8)$ were incubated for $24 \mathrm{~h}$ with
increasing concentrations of a) cyclosporine A, b)
tacrolimus (FK506), c) methylprednisolone, d)
mycophenolate mofetil or e) everolimus. Serum-free
RPMI served as control. Cell growth was assessed by
$\left[{ }^{3} \mathrm{H}\right]$-thymidine incorporation and values are presented
as mean \pm sEM of independent experiments performed in
eight different cell lines, expressed as relative
proliferation compared with the control (serum-free
RPMI). ${ }^{*}: \mathrm{p}<0.05$.

myofibroblasts in our in vitro model. But as this effect was accompanied by decreased cell viability [27], these concentrations were not further pursued. Even if tissue concentrations of CsA and FK506 are considerably higher than blood concentrations, it is very unlikely that these high tissue concentrations would be reached in the lung, since accumulation of these lipophilic drugs mainly occurs in lipid-rich organs [35]. It is important to note that lung tissue concentrations are expected to increase by additional inhalation of CsA, and this new form of administration provided an advantage of survival over conventional therapy alone in lung transplant recipients with bronchiolitis obliterans [36]. Although we observed a similar pro-proliferative effect of CsA and FK506 when used in clinically relevant concentrations in our in vitro model, clinical data demonstrated FK506 to be superior to CsA with regard to freedom from BOS after lung transplantation [37], and course of lung function in lung-transplanted patients with BOS [15]. However, there is no comparable data for HSCT patients.

In contrast to the tested calcineurin inhibitors, mPRED exerted a significant growth-inhibitory effect at concentrations of 10,50 and $100 \mathrm{mg} \cdot \mathrm{L}^{-1}$. These concentrations might be reached by steroid pulses of 500-1000 mg per day [38], as might be administered initially after diagnosis of bronchiolitis obliterans. The observed antiproliferative effect of mPRED in our in vitro model is in line with data presented previously $[39,40]$. Our in vitro data reflect the clinical experience that high-dose i.v. mPRED may cause a favourable 
FIGURE 5 Effect of combination treatment with tacrolimus (FK506), mycophenolate mofetil (MMF) and methylprednisolone (mPRED), and of MMF alone on myofibroblast proliferation compared with serum-free RPMI. Primary human lung myofibroblasts were incubated for $24 \mathrm{~h}$ with FK506 $\left(0.01 \mathrm{mg} \cdot \mathrm{L}^{-1}\right), \mathrm{MMF}\left(1 \mathrm{mg} \cdot \mathrm{L}^{-1}\right)$ and $\mathrm{mPRED}\left(10 \mathrm{mg} \cdot \mathrm{L}^{-1}\right)$ or with MMF $\left(1 \mathrm{mg} \cdot \mathrm{L}^{-1}\right)$ alone. Serum-free RPMI served as control. Cell growth was assessed by $\left[{ }^{3} \mathrm{H}\right]$ thymidine incorporation and values are presented as mean \pm SEM of independent experiments, expressed as relative proliferation compared with the control (serum-free RPMI).

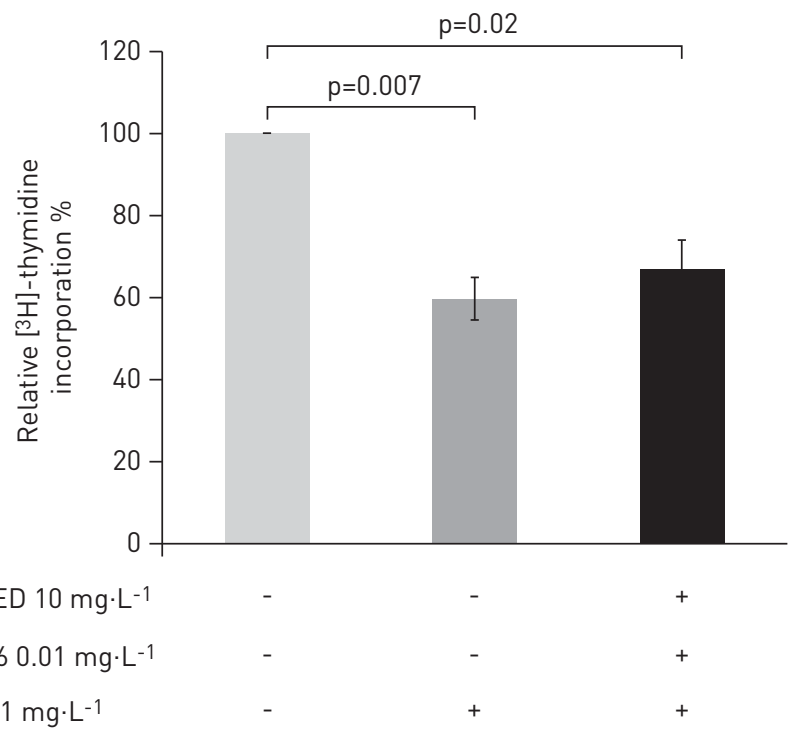

response in bronchiolitis obliterans after bone marrow transplantation [41]. In contrast, patients with isolated lymphocytic bronchiolitis after lung transplantation did not show any improvement in FEV1 after pulsed mPRED treatment [42].

Similar to mPRED, MMF caused a significant inhibition of cell proliferation, and this is in line with previously published in vitro data $[39,43,44]$. The concentration of $1 \mathrm{mg} \cdot \mathrm{L}^{-1} \mathrm{MMF}$ corresponds to the target whole-blood level for transplant patients [45]. MMF has been demonstrated to be safe and effective when used as first-line immunosuppression after lung transplantation [46], and conversion from calcineurin inhibitors to MMF in patients with BOS after lung transplantation led to a stabilisation of graft function in some patients [47]. Furthermore, in a prospective trial comparing MMF with azathioprine in lung transplant recipients it could be demonstrated that MMF significantly reduced graft loss due to BOS and tended to improve overall survival [48].

Everolimus, an inhibitor of the mammalian target of rapamycin, had no significant effect on cell proliferation in our in vitro model. An antiproliferative effect of everolimus has been described earlier in haematopoietic and non-haematopoietic cells $[49,50]$. However, in these studies everolimus only inhibited growth factor-triggered proliferation $[49,50]$, whereas we looked at the effect of the drug in a FCS-/growthfactor-free cell culture medium. Even though data from lung transplantation demonstrated a significant
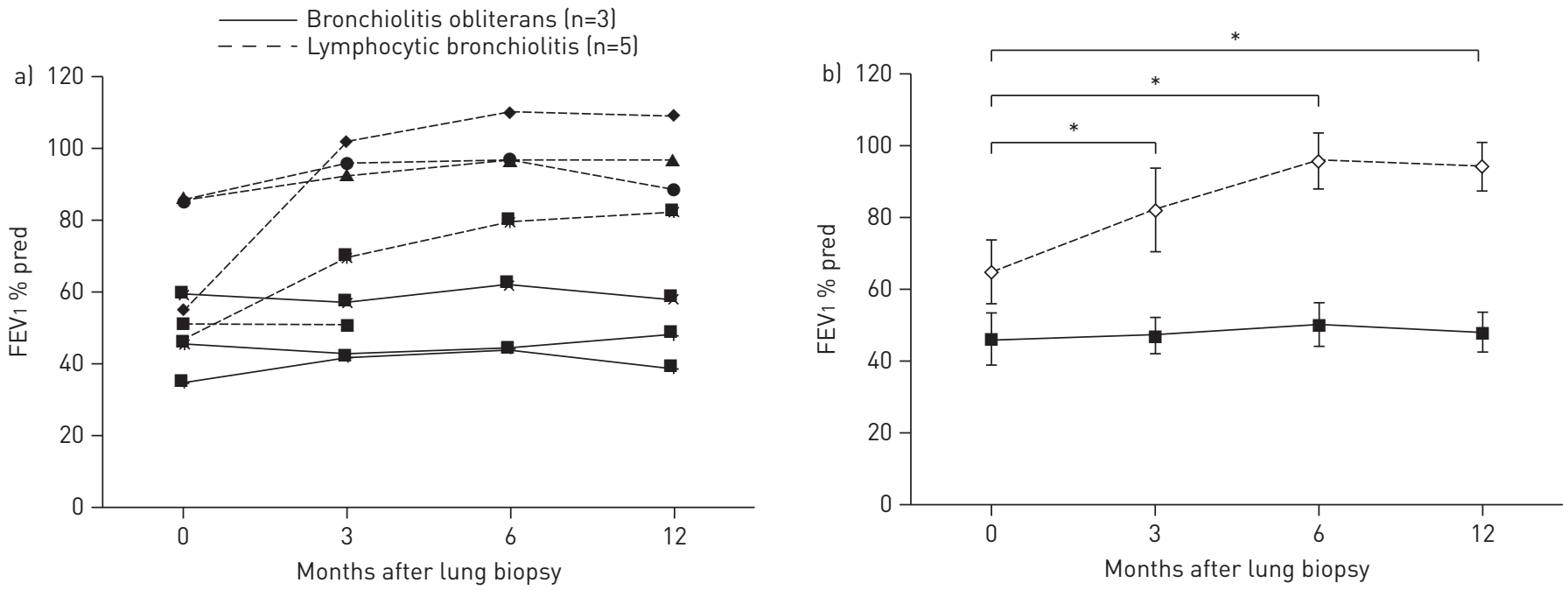

FIGURE 6 a) Forced expiratory volume in $1 \mathrm{~s}$ (FEV1) \% predicted of all eight patients; b) mean FEV1 \% pred values of the patient group with histologically confirmed bronchiolitis obliterans $(n=3)$ and of the patient group with lymphocytic bronchiolitis $(n=5)$. Time points show video-assisted thoracoscopic surgery and 3 months, 6 months and 12 months after surgery. ${ }^{\star}: p \leqslant 0.05$. 
reduction in the rate of the decline in lung function under everolimus treatment [51], no such data exist for BOS after HSCT. Finally, everolimus should be used with caution due to potential significant lung and liver toxicities [52-55].

After diagnosis of bronchiolitis obliterans/lymphocytic bronchiolitis, all eight patients in this study group were treated with a triple therapy comprising mPRED, FK506 and MMF. Therefore, we also tested the effect of this combination therapy in our in vitro model and the combination of mPRED and MMF was able to fully antagonise the pro-proliferative potential of FK506. Importantly, this effect might be attributed mainly to $\mathrm{MMF}$, as monostimulation with MMF exhibited a similar antiproliferative effect as compared to the triple stimulation. The observed in vitro growth-inhibitory effect of MMF further substantiates the potential power of the drug to limit the progression of fibroproliferative diseases, and underlines the need for further clinical studies with this drug. We are aware that our in vitro model does not fully represent the multiple effects between different cell types and soluble factors resulting in bronchiolitis obliterans in vivo. However, considering our in vitro data, the role of calcineurin inhibitors in the treatment of bronchiolitis obliterans after HSCT should at least be questioned, and MMF-based (calcineurin inhibitor-free) regimens may be further evaluated, including a combination of MMF with azithromycin as a prophylaxis, the latter has been shown to be able to reduce BOS after lung transplantation [56]. To date, only one randomised trial studied the effect of MMF in the initial treatment of chronic GvHD [57]. However, since patients with BOS were excluded from this trial and numbers of newly diagnosed BOS were low, no conclusion concerning the effect of MMF effect on BOS could be drawn.

Finally, analysis of serial follow-up pulmonary function tests after lung biopsy and under the aforementioned triple immunosuppressive therapy revealed that patients with histological lymphocytic bronchiolitis experienced a statistically relevant improvement of FEV1 \% pred, whereas in patients with histologically confirmed bronchiolitis obliterans FEV1 \% pred remained unchanged, and this is in line with the findings from a larger series [24]. This clinical observation supports our in vitro data showing an antiproliferative effect of the combination therapy with mPRED, MMF and FK506. However, this beneficial effect seems to occur predominantly in patients with lymphocytic bronchiolitis, but not in those with bronchiolitis obliterans, and thus indicates that only in conditions with considerable inflammatory cell infiltrate can the progression of disease be delayed, whereas with only scant inflammation but pronounced fibrosis the potential benefit might be limited. Importantly, neutrophilia of $>15 \%$ in the BAL fluid has been demonstrated to be predictive of FEV1 response to azithromycin in patients with BOS after lung transplantation [58], whereas patients without neutrophilia in the BAL benefited from the addition of the leukotriene receptor antagonist montelukast [59]. Transferring these findings to our study group, one would add azithromycin to the immunosuppressive regimen in those patients with histological lymphocytic bronchiolitis and relevant BAL neutrophilia, and additional montelukast for those patients with low neutrophilic lymphocytic bronchiolitis. Recent data obtained from HSCT patients with BOS demonstrated that the combination of azithromycin and montelukast permitted a reduction of corticosteroid exposure [60], but lung function values did not improve under azithromycin treatment in a randomised placebocontrolled study [61]. In contrast, combining standard immunosuppressive therapy with montelukast caused an improvement in pulmonary function test values in patients with chronic GvHD [62].

We acknowledge that the present study has some limitations. Firstly, we used drug concentrations which are in the range of target whole-blood levels, but may not reflect lung tissue concentrations. However, no representative data exist on tissue concentrations of the studied drugs. Furthermore, our in vitro model rather resembles a constant infusion of the drug, in contrast to the peak and trough levels of the in vivo situation. Secondly, the mechanism by which the calcineurin inhibitors exert their pro-proliferative effect was not addressed, but this would have exceeded the scope of this manuscript. Thirdly, the number of patients studied is small. Since bronchiolitis obliterans is a rare disease and few patients undergo lung biopsy, it will be a big challenge to get larger patient numbers. However, given the uniformity of the results, we are confident that our data are representative for this type of experiments. Finally, we are aware that the transition of findings obtained by in vitro models to clinical practice has to be done with caution, and controlled clinical trials are needed to test basic research data.

\section{Conclusion}

Our data showed that CsA and FK506 have a pro-proliferative effect on primary human lung myofibroblasts obtained from patients with BOS after allogeneic HSCT, and their role in the treatment of bronchiolitis obliterans after HSCT might be questioned. However, combination of FK506, mPRED and MMF significantly inhibited cell proliferation in vitro, which was mainly due to the growth-inhibitory effect of MMF. Our findings suggest that in patients with bronchiolitis obliterans after HSCT MMF-containing, calcineurin inhibitor-free regimens should be further evaluated. 


\section{References}

1 Ljungman P, Bregni M, Brune M, et al. Allogeneic and autologous transplantation for haematological diseases, solid tumours and immune disorders: current practice in Europe 2009. Bone Marrow Transplant 2010; 45: 219-234. Copelan EA. Hematopoietic stem-cell transplantation. N Engl J Med 2006; 354: 1813-1826.

3 Filipovich AH, Weisdorf D, Pavletic S, et al. National Institutes of Health consensus development project on criteria for clinical trials in chronic graft-versus-host disease: I. Diagnosis and staging working group report. Biol Blood Marrow Transplant 2005; 11: 945-956.

4 Chien JW, Martin PJ, Flowers ME, et al. Implications of early airflow decline after myeloablative allogeneic stem cell transplantation. Bone Marrow Transplant 2004; 33: 759-764.

5 Philit F, Wiesendanger T, Archimbaud E, et al. Post-transplant obstructive lung disease ("bronchiolitis obliterans"): a clinical comparative study of bone marrow and lung transplant patients. Eur Respir J 1995; 8: 551-558.

6 Hildebrandt GC, Fazekas T, Lawitschka A, et al. Diagnosis and treatment of pulmonary chronic GVHD: report from the consensus conference on clinical practice in chronic GVHD. Bone Marrow Transplant 2011; 46: $1283-1295$.

7 Williams KM, Chien JW, Gladwin MT, et al. Bronchiolitis obliterans after allogeneic hematopoietic stem cell transplantation. JAMA 2009; 302: 306-314.

8 Chien JW, Duncan S, Williams KM, et al. Bronchiolitis obliterans syndrome after allogeneic hematopoietic stem cell transplantation - an increasingly recognized manifestation of chronic graft-versus-host disease. Biol Blood Marrow Transplant 2010; 16: Suppl. 1, S106-S114.

9 Duncker C, Dohr D, Harsdorf S, et al. Non-infectious lung complications are closely associated with chronic graftversus-host disease: a single center study of incidence, risk factors and outcome. Bone Marrow Transplant 2000; 25 : $1263-1268$.

10 Yoshihara S, Yanik G, Cooke KR, et al. Bronchiolitis obliterans syndrome (BOS), bronchiolitis obliterans organizing pneumonia (BOOP), and other late-onset noninfectious pulmonary complications following allogeneic hematopoietic stem cell transplantation. Biol Blood Marrow Transplant 2007; 13: 749-759.

11 Yousem SA. The histological spectrum of pulmonary graft-versus-host disease in bone marrow transplant recipients. Hum Pathol 1995; 26: 668-675.

12 Marras TK, Chan CK, Lipton JH, et al. Long-term pulmonary function abnormalities and survival after allogeneic marrow transplantation. Bone Marrow Transplant 2004; 33: 509-517.

13 Clark JG, Crawford SW, Madtes DK, et al. Obstructive lung disease after allogeneic marrow transplantation. Clinical presentation and course. Ann Intern Med 1989; 111: 368-376.

14 Snell GI, Esmore DS, Williams TJ. Cytolytic therapy for the bronchiolitis obliterans syndrome complicating lung transplantation. Chest 1996; 109: 874-878.

15 Cairn J, Yek T, Banner NR, et al. Time-related changes in pulmonary function after conversion to tacrolimus in bronchiolitis obliterans syndrome. J Heart Lung Transplant 2003; 22: 50-57.

16 Dudek AZ, Mahaseth H, DeFor TE, et al. Bronchiolitis obliterans in chronic graft-versus-host disease: analysis of risk factors and treatment outcomes. Biol Blood Marrow Transplant 2003; 9: 657-666.

17 Sakaida E, Nakaseko C, Harima A, et al. Late-onset noninfectious pulmonary complications after allogeneic stem cell transplantation are significantly associated with chronic graft-versus-host disease and with the graft-versusleukemia effect. Blood 2003; 102: 4236-4242.

18 Kotloff RM, Ahya VN, Crawford SW. Pulmonary complications of solid organ and hematopoietic stem cell transplantation. Am J Respir Crit Care Med 2004; 170: 22-48.

19 Hanssen L, Frye BC, Ostendorf T, et al. Y-box binding protein-1 mediates profibrotic effects of calcineurin inhibitors in the kidney. J Immunol 2011; 187: 298-308.

20 Ninova D, Covarrubias M, Rea DJ, et al. Acute nephrotoxicity of tacrolimus and sirolimus in renal isografts: differential intragraft expression of transforming growth factor- $\beta 1$ and $\alpha$-smooth muscle actin. Transplantation 2004; 78: 338-344.

21 Shihab FS, Andoh TF, Tanner AM, et al. Sodium depletion enhances fibrosis and the expression of TGF- $\beta 1$ and matrix proteins in experimental chronic cyclosporine nephropathy. Am J Kidney Dis 1997; 30: 71-81.

22 Sobral LM, Aseredo F, Agostini M, et al. Molecular events associated with ciclosporin A-induced gingival overgrowth are attenuated by Smad7 overexpression in fibroblasts. J Periodontal Res 2012; 47: 149-158.

23 Hunt J, Cheng A, Hoyles A, et al. Cyclosporin A has direct effects on adult neural precursor cells. J Neurosci 2010; 30: $2888-2896$

24 Holbro A, Lehmann T, Girsberger S, et al. Lung histology predicts outcome of bronchiolitis obliterans syndrome after hematopoietic stem cell transplantation. Biol Blood Marrow Transplant 2013; 19: 973-980.

25 Quanjer PH, Tammeling GJ, Cotes JE, et al. Lung volumes and forced ventilatory flows. Report Working Party Standardization of Lung Function Tests, Official Statement of the European Respiratory Society. Eur Respir J 1993; 6: Suppl. 16, 5-40.

26 Tamm M, Roth M, Malouf M, et al. Primary fibroblast cell cultures from transbronchial biopsies of lung transplant recipients. Transplantation 2001; 71: 337-339.

27 Hostettler KE, Roth M, Burgess JK, et al. Cyclosporine A mediates fibroproliferation through epithelial cells. Transplantation 2004; 77: 1886-1893.

28 Santo Tomas LH, Loberiza FR Jr, Klein JP, et al. Risk factors for bronchiolitis obliterans in allogeneic hematopoietic stem-cell transplantation for leukemia. Chest 2005; 128: 153-161.

29 Beschorner WE, Saral R, Hutchins GM, et al. Lymphocytic bronchitis associated with graft-versus-host disease in recipients of bone-marrow transplants. N Engl J Med 1978; 299: 1030-1036.

30 Boehler A, Chamberlain D, Kesten S, et al. Lymphocytic airway infiltration as a precursor to fibrous obliteration in a rat model of bronchiolitis obliterans. Transplantation 1997; 64: 311-317.

31 Nakamoto-Matsubara R, Nishikii H, Yamada K, et al. Early pathologic findings of bronchiolitis obliterans after allogeneic hematopoietic stem cell transplantation: a proposal from a case. Case Report Hematol 2012; 2012: 957612.

32 Briffa N, Morris RE. New immunosuppressive regimens in lung transplantation. Eur Respir J 1997; 10: 2630-2637. 
Aisa Y, Mori T, Nakazato T, et al. Effects of immunosuppressive agents on magnesium metabolism early after allogeneic hematopoietic stem cell transplantation. Transplantation 2005; 80: 1046-1050.

34 Mihatsch MJ, Kyo M, Morozumi K, et al. The side-effects of ciclosporine-A and tacrolimus. Clin Nephrol 1998; 49: 356-363.

35 Lensmeyer GL, Wiebe DA, Carlson IH, et al. Concentrations of cyclosporin A and its metabolites in human tissues postmortem. J Anal Toxicol 1991; 15: 110-115.

36 Iacono AT, Corcoran TE, Griffith BP, et al. Aerosol cyclosporin therapy in lung transplant recipients with bronchiolitis obliterans. Eur Respir J 2004; 23: 384-390.

37 Hachem RR, Yusen RD, Chakinala MM, et al. A randomized controlled trial of tacrolimus versus cyclosporine after lung transplantation. J Heart Lung Transplant 2007; 26: 1012-1018.

38 Behar-Cohen FF, Gauthier S, El Aouni A, et al. Methylprednisolone concentrations in the vitreous and the serum after pulse therapy. Retina 2001; 21: 48-53.

39 Azzola A, Havryk A, Chhajed P, et al. Everolimus and mycophenolate mofetil are potent inhibitors of fibroblast proliferation after lung transplantation. Transplantation 2004; 77: 275-280.

40 Stewart AG, Fernandes D, Tomlinson PR. The effect of glucocorticoids on proliferation of human cultured airway smooth muscle. Br J Pharmacol 1995; 116: 3219-3226.

41 Ratjen F, Rjabko O, Kremens B. High-dose corticosteroid therapy for bronchiolitis obliterans after bone marrow transplantation in children. Bone Marrow Transplant 2005; 36: 135-138.

42 Ross DJ, Marchevsky A, Kramer M, et al. "Refractoriness" of airflow obstruction associated with isolated lymphocytic bronchiolitis/bronchitis in pulmonary allografts. J Heart Lung Transplant 1997; 16: 832-838.

43 Heinz C, Hudde T, Heise K, et al. Antiproliferative effect of mycophenolate mofetil on cultured human Tenon fibroblasts. Graefes Arch Clin Exp Ophthalmol 2002; 240: 408-414.

44 Badid C, Vincent M, McGregor B, et al. Mycophenolate mofetil reduces myofibroblast infiltration and collagen III deposition in rat remnant kidney. Kidney Int 2000; 58: 51-61.

45 Tredger JM, Brown NW, Adams J, et al. Monitoring mycophenolate in liver transplant recipients: toward a therapeutic range. Liver Transpl 2004; 10: 492-502.

46 McNeil K, Glanville AR, Wahlers TK, et al. Comparison of mycophenolate mofetil and azathioprine for prevention of bronchiolitis obliterans syndrome in de novo lung transplant recipients. Transplantation 2006; 81: 998-1003.

47 Groetzner J, Wittwer T, Kaczmarek I, et al. Conversion to sirolimus and mycophenolate can attenuate the progression of bronchiolitis obliterans syndrome and improves renal function after lung transplantation. Transplantation 2006; 81: 355-360.

48 Speich R, Schneider S, Hofer M, et al. Mycophenolate mofetil reduces alveolar inflammation, acute rejection and graft loss due to bronchiolitis obliterans syndrome after lung transplantation. Pulm Pharmacol Ther 2010; 23: 445-449.

49 Schuler W, Sedrani R, Cottens S, et al. SDZ RAD, a new rapamycin derivative: pharmacological properties in vitro and in vivo. Transplantation 1997; 64: 36-42.

50 Böhler $\mathrm{T}$, Waiser J, Budde $\mathrm{K}$, et al. The in vivo effect of rapamycin derivative SDZ RAD on lymphocyte proliferation. Transplant Proc 1998; 30: 2195-2197.

51 Snell GI, Valentine VG, Vitulo P, et al. Everolimus versus azathioprine in maintenance lung transplant recipients: an international, randomized, double-blind clinical trial. Am J Transplant 2006; 6: 169-177.

52 Pham PT, Pham PC, Danovitch GM, et al. Sirolimus-associated pulmonary toxicity. Transplantation 2004; 77: $1215-1220$

53 Vandewiele B, Vandecasteele SJ, Vanwalleghem L, et al. Diffuse alveolar hemorrhage induced by everolimus. Chest 2010; 137: 456-459.

54 Champion L, Stern M, Israël-Biet D, et al. Brief communication: sirolimus-associated pneumonitis: 24 cases in renal transplant recipients. Ann Intern Med 2006; 144: 505-509.

55 Platzbecker U, von Bonin M, Goekkurt E, et al. Graft-versus-host disease prophylaxis with everolimus and tacrolimus is associated with a high incidence of sinusoidal obstruction syndrome and microangiopathy: results of the EVTAC trial. Biol Blood Marrow Transplant 2009; 15: 101-108.

56 Vos R, Vanaudenaerde BM, Verleden SE, et al. A randomised controlled trial of azithromycin to prevent chronic rejection after lung transplantation. Eur Respir J 2011; 37: 164-172.

57 Martin PJ, Storer BE, Rowley SD, et al. Evaluation of mycophenolate mofetil for initial treatment of chronic graftversus-host disease. Blood 2009; 113: 5074-5082.

58 Verleden GM, Vanaudenaerde BM, Dupont LJ, et al. Azithromycin reduces airway neutrophilia and interleukin-8 in patients with bronchiolitis obliterans syndrome. Am J Respir Crit Care Med 2006; 174: 566-570.

59 Verleden GM, Verleden SE, Vos R, et al. Montelukast for bronchiolitis obliterans syndrome after lung transplantation: a pilot study. Transpl Int 2011; 24: 651-656.

60 Norman BC, Jacobsohn DA, Williams KM, et al. Fluticasone, azithromycin and montelukast therapy in reducing corticosteroid exposure in bronchiolitis obliterans syndrome after allogeneic hematopoietic SCT: a case series of eight patients. Bone Marrow Transplant 2011; 46: 1369-1373.

61 Lam DC, Lam B, Wong MK, et al. Effects of azithromycin in bronchiolitis obliterans syndrome after hematopoietic SCT - a randomized double-blinded placebo-controlled study. Bone Marrow Transplant 2011; 46: 1551-1556.

62 Or R, Gesundheit B, Resnick I, et al. Sparing effect by montelukast treatment for chronic graft versus host disease: a pilot study. Transplantation 2007; 83: 577-581. 